\title{
SpacePy - A Python-based Library of Tools for the Space Sciences
}

\author{
Steven K. Morley ${ }^{\ddagger *}$, Daniel T. Welling ${ }^{\ddagger}$, Josef Koller ${ }^{\ddagger}$, Brian A. Larsen ${ }^{\ddagger}$, Michael G. Henderson ${ }^{\ddagger}$, Jonathan Niehof
}

\begin{abstract}
Space science deals with the bodies within the solar system and the interplanetary medium; the primary focus is on atmospheres and above-at Earth the short timescale variation in the the geomagnetic field, the Van Allen radiation belts and the deposition of energy into the upper atmosphere are key areas of investigation.

SpacePy is a package for Python, targeted at the space sciences, that aims to make basic data analysis, modeling and visualization easier. It builds on the capabilities of the well-known NumPy and matplotlib packages. Publication quality output direct from analyses is emphasized. The SpacePy project seeks to promote accurate and open research standards by providing an open environment for code development. In the space physics community there has long been a significant reliance on proprietary languages that restrict free transfer of data and reproducibility of results. By providing a comprehensive library of widely-used analysis and visualization tools in a free, modern and intuitive language, we hope that this reliance will be diminished for non-commercial users.

SpacePy includes implementations of widely used empirical models, statistical techniques used frequently in space science (e.g. superposed epoch analysis), and interfaces to advanced tools such as electron drift shell calculations for radiation belt studies. SpacePy also provides analysis and visualization tools for components of the Space Weather Modeling Framework including streamline tracing in vector fields. Further development is currently underway. External libraries, which include well-known magnetic field models, high-precision time conversions and coordinate transformations are accessed from Python using ctypes and f2py. The rest of the tools have been implemented directly in Python.

The provision of open-source tools to perform common tasks will provide openness in the analysis methods employed in scientific studies and will give access to advanced tools to all space scientists, currently distribution is limited to non-commercial use.
\end{abstract}

Index Terms-astronomy, atmospheric science, space weather, visualization

\section{Introduction}

For the purposes of this article we define space science as the study of the plasma environment of the solar system. That is, the Earth and other planets are all immersed in the Sun's tenuous outer atmosphere (the heliosphere), and all are affected in some way by natural variations in the Sun. This is of particular importance at Earth where the magnetized plasma flowing out from the Sun interacts with Earth's magnetic field and can affect technological systems and climate. The primary focus here is on planetary atmospheres and above - at Earth the short timescale variation in

* Corresponding author: smorley@lanl.gov

* Los Alamos National Laboratory

Copyright $(02010$ Steven K. Morley et al. This is an open-access article distributed under the terms of the Creative Commons Attribution License, which permits unrestricted use, distribution, and reproduction in any medium, provided the original author and source are credited. the the geomagnetic field, the Van Allen radiation belts [Mor10] and the deposition of energy into the upper atmosphere [Mly10] are key areas of investigation.

SpacePy was conceived to provide a convenient library for common tasks in the space sciences. A number of routine analyses used in space science are much less common in other fields (e.g. superposed epoch analysis) and modules to perform these analyses are provided. This article describes the initial release of SpacePy (0.1.0), available from Los Alamos National Laboratory. at http://spacepy.lanl.gov. Currently SpacePy is available on a noncommercial research license, but open-sourcing of the software is in process.

\section{SpacePy organization}

As packages such as NumPy, SciPy and matplotlib have become de facto standards in Python, we have adopted these as the prerequisites for SpacePy.

The SpacePy package provides a number of modules, for a variety of tasks, which will be briefly described in this article. HTML help for SpacePy is generated using epydoc and is bundled with the package. This can be most easily accessed on import of SpacePy (or any of its modules) by running the help() function in the appropriate namespace. A schematic of the organization of SpacePy is shown in figure 1. In this article we will describe the core modules of SpacePy and provide some short examples of usage and output.

The most general of the bundled modules is Toolbox. At the time of writing this contains (among others): a convenience function for graphically displaying the contents of dictionaries recursively; windowing mean calculations; optimal bin width estimation for histograms via the Freedman-Diaconis method; an update function to fetch the latest OMNI (solar wind/geophysical index) database and leap-second list; comparison of two time series for overlap or common elements.

The other modules have more specific aims and are primarily based on new classes. Time provides a container class for times in a range of time systems, conversion between those systems and extends the functionality of datetime for space science use. Coordinates provides a class, and associated functions, for the handling of coordinates and transformations between common coordinate systems. IrbemPy is a module that wraps the IRBEM magnetic field library. Radbelt implements a 1-D radial diffusion code along with diffusion coefficient calculations and plotting routines. SeaPy provides generic one- and two-dimensional superposed epoch analysis classes and some plotting and statistical testing 


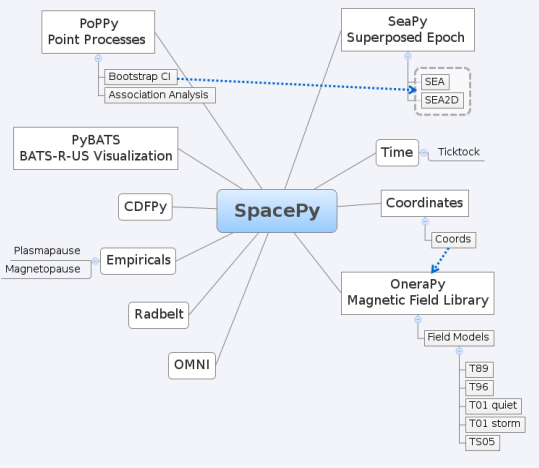

Fig. 1: A schematic of the organization and contents of the SpacePy package at the time of writing.

for superposed epoch analysis. PoPPy is a module for analysis of point processes, in particular it provides association analysis tools. Empiricals provides implementations of some common empirical models such as plasmapause and magnetopause locations. PyBATS is an extensive sub-package providing tools for the convenient reading, writing and display of output from the Space Weather Modeling Framework (a collection of coupled models of the SunEarth system). $P y C D F$ is a fully object-oriented interface to the NASA Common Data Format library.

\section{Time conversions}

SpacePy provides a time module that enables convenient manipulation of times and conversion between time systems commonly used in space sciences:

\section{1) NASA Common Data Format (CDF) epoch}

2) International Atomic Time (TAI)

3) Coordinated Universal Time (UTC)

4) Gregorian ordinal time (RDT)

5) Global Positioning System (GPS) time

6) Julian day (JD)

7) modified Julian day (MJD)

8) day of year (DOY)

9) elapsed days of year (eDOY)

10) UNIX time (UNX)

This is implemented as a container class built on the functionality of the core Python datetime module. To illustrate its use, we present code which instantiates a Ticktock object, and fetches the time in different systems:

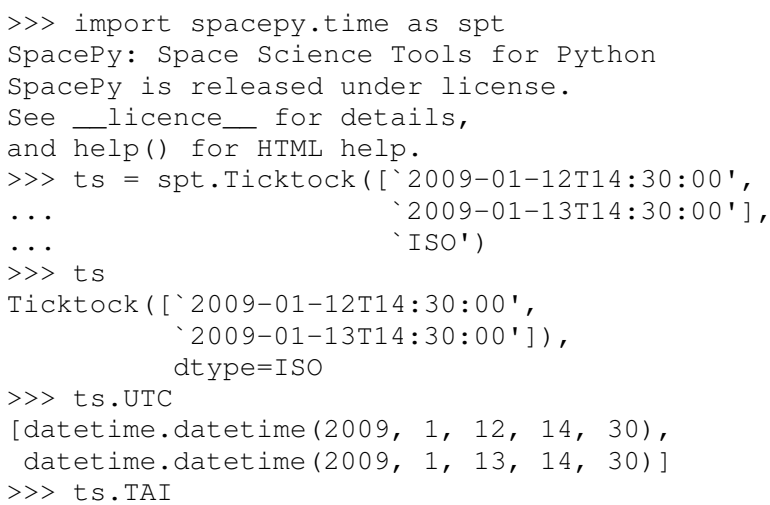

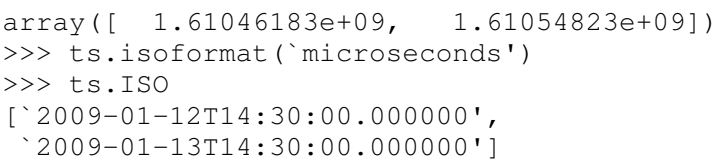

\section{Coordinate handling}

Coordinate handling and conversion is performed by the coordinates module. This module provides the Coords class for coordinate data management. Transformations between cartesian and spherical coordinates are implemented directly in Python, but the coordinate conversions are currently handled as calls to the IRBEM library.

In the following example two locations are specified in a geographic cartesian coordinate system and converted to spherical coordinates in the geocentric solar magnetospheric (GSM) coordinate system. The coordinates are stored as object attributes. For coordinate conversions times must be supplied as many of the coordinate systems are defined with respect to, e.g., the position of the Sun, or the plane of the Earth's dipole axis, which are timedependent.

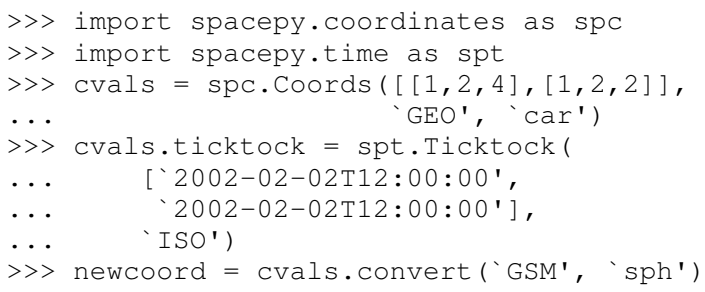

A new, higher-precision $\mathrm{C}$ library to perform time conversions, coordinate conversions, satellite ephemeris calculations, magnetic field modeling and drift shell calculations-the LANLGeoMag (LGM) library-is currently being wrapped for Python and will eventually replace the IRBEM library as the default in SpacePy.

\section{The IRBEM library}

ONERA (Office National d'Etudes et Recherches Aerospatiales) provide a FORTRAN library, the IRBEM library [Bos07], that provides routines to compute magnetic coordinates for any location in the Earth's magnetic field, to perform coordinate conversions, to compute magnetic field vectors in geospace for a number of external field models, and to propagate satellite orbits in time.

A number of key routines in the IRBEM library have been wrapped uing f2py, and a 'thin layer' module IrbemPy has been written for easy access to these routines. Current functionality includes calls to calculate the local magnetic field vectors at any point in geospace, calculation of the magnetic mirror point for a particle of a given pitch angle (the angle between a particle's velocity vector and the magnetic field line that it immediately orbits such that a pitch angle of 90 degrees signifies gyration perpendicular to the local field) anywhere in geospace, and calculation of electron drift shells in the inner magnetosphere.

As mentioned in the description of the Coordinates module, access is also provided to the coordinate transformation capabilities of the IRBEM library. These can be called directly, but IrbemPy is easier to work with using Coords objects. This is by design as we aim to incorporate the LGM library and replace the calls to IRBEM with calls to LGM without any change to the Coordinates syntax. 


\section{OMNI}

The OMNI database [Kin05] is an hourly resolution, multi-source data set with coverage from November 1963; higher temporal resolution versions of the OMNI database exist, but with coverage from 1995. The primary data are near-Earth solar wind, magnetic field and plasma parameters. However, a number of modern magnetic field models require derived input parameters, and [Qin07] have used the publicly-available OMNI database to provide a modified version of this database containing all parameters necessary for these magnetic field models. These data are currently updated and maintained by Dr. Bob Weigel and are available through ViRBO (Virtual Radiation Belt Observatory) ${ }^{1}$.

In SpacePy this data is made available on request on install; if not downloaded when SpacePy is installed and attempt to import the omni module will ask the user whether they wish to download the data. Should the user require the latest data, the update function within spacepy.toolbox can be used to fetch the latest files from ViRBO.

As an example, we fetch the OMNI data for the powerful "Hallowe'en" storms of October and November, 2003. These geomagnetic storms were driven by two solar coronal mass ejections that reached the Earth on October 29th and November 20th.

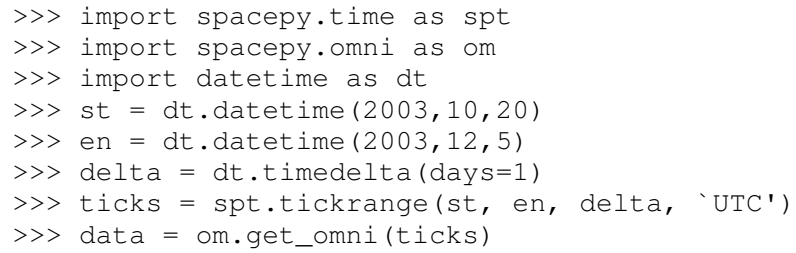

data is a dictionary containing all the OMNI data, by variable, for the timestamps contained within the Ticktock object ticks

\section{Superposed Epoch Analysis}

Superposed epoch analysis is a technique used to reveal consistent responses, relative to some repeatable phenomenon, in noisy data [Chr08]. Time series of the variables under investigation are extracted from a window around the epoch and all data at a given time relative to epoch forms the sample of events at that lag. The data at each time lag are then averaged so that fluctuations not consistent about the epoch cancel. In many superposed epoch analyses the mean of the data at each time $u$ relative to epoch, is used to represent the central tendency. In SeaPy we calculate both the mean and the median, since the median is a more robust measure of central tendency and is less affected by departures from normality. SeaPy also calculates a measure of spread at each time relative to epoch when performing the superposed epoch analysis; the interquartile range is the default, but the median absolute deviation and bootstrapped confidence intervals of the median (or mean) are also available. The output of the example below is shown in figure 2 .

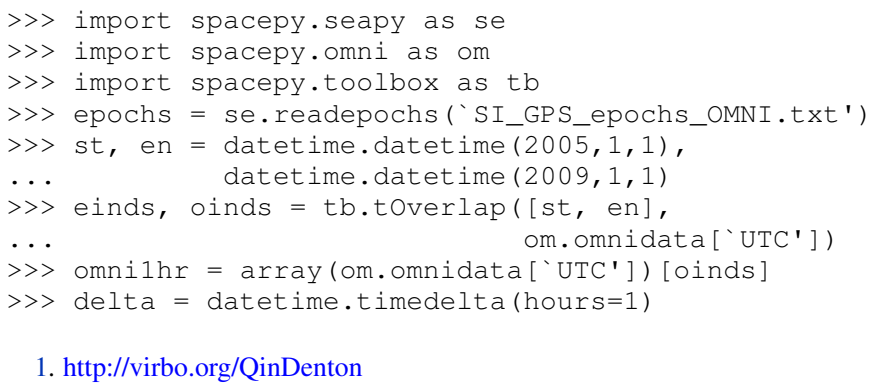

1. http://virbo.org/QinDenton

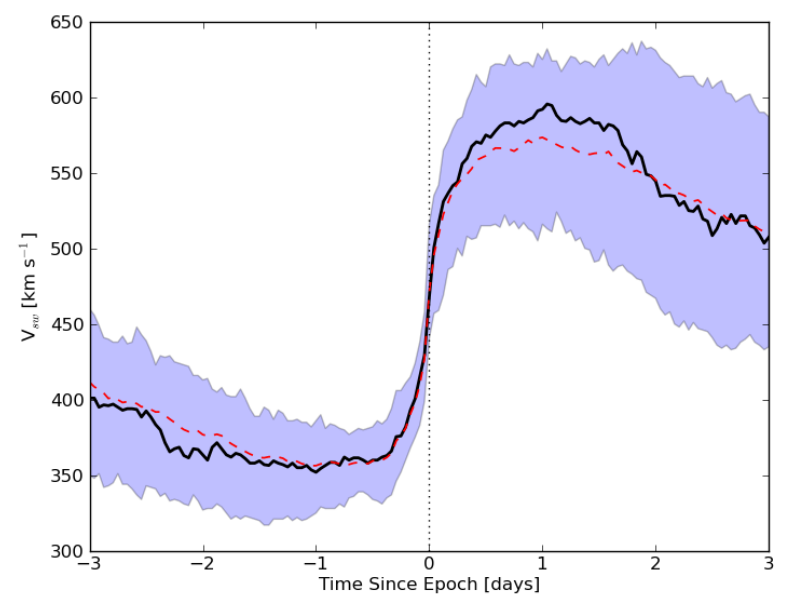

Fig. 2: A typical output from the SpacePy Sea class using OMNI solar wind velocity data. The black line marks the superposed epoch median, the red dashed line marks the superposed epoch mean, and the blue fill marks the interquartile range. This figure was generated using the code in the text and a list of 67 events published by [Mor10].

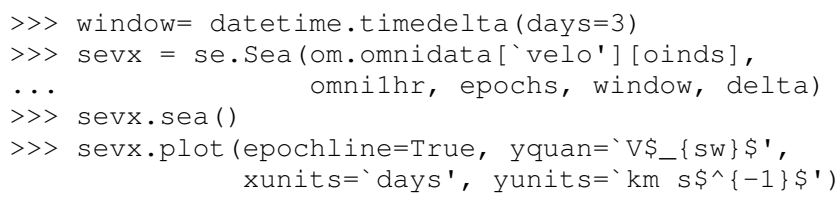

More advanced features of this module have been used in analyses of the Van Allen radiation belts and can be found in the peerreviewed literature [Mor10].

\section{Association analysis}

This module provides a point process class P P ro and methods for association analysis (see, e.g., [Mor07]). This module is intended for application to discrete time series of events to assess statistical association between the series and to calculate confidence limits. Since association analysis is rather computationally expensive, this example shows timing. To illustrate its use, we here reproduce the analysis of [Wil09] using SpacePy. After importing the necessary modules, and assuming the data has already been loaded, P P ro objects are instantiated. The association analysis is performed by calling the assoc method and bootstrapped confidence intervals are calculated using the aa_ci method. It should be noted that this type of analysis is computationally expensive and, though currently implemented in pure Python may be rewritten using Cython or $\mathrm{C}$ to gain speed.

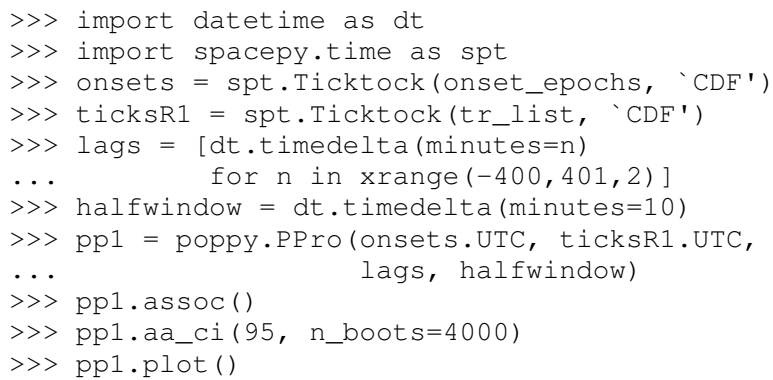

The output is shown in figure 3 and can be compared to figure $6 a$ of [Wil09]. 


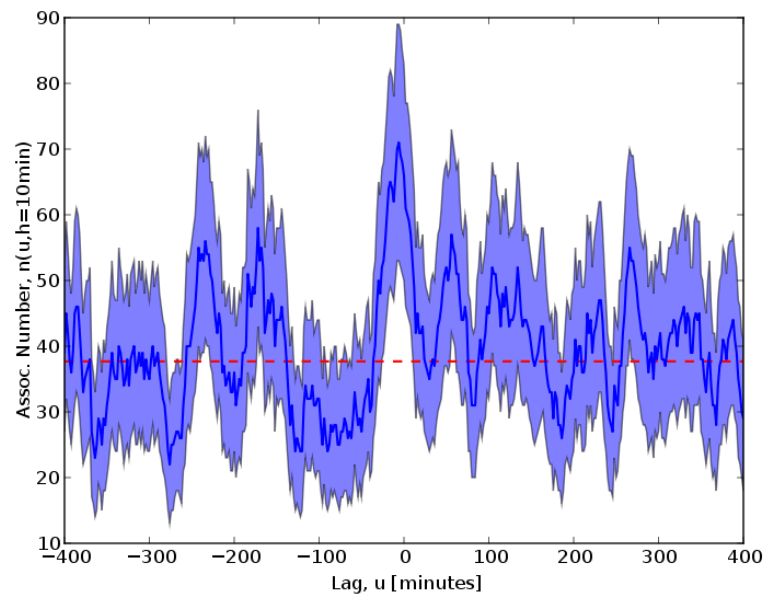

Fig. 3: Reproduction of the association analysis done by [Wil09], using the PoPPy module of SpacePy. The figure shows a significant association around zero time lag between the two point processes under study (northward turnings of the interplanetary magnetic field and auroral substorm onsets).

\section{NASA Common Data Format}

At the time of writing, limited support for NASA $\mathrm{CDF}^{2}$ has been written in to SpacePy. NASA themselves have worked with the developers of both IDL ${ }^{\mathrm{TM}}$ and MatLab ${ }^{\mathrm{TM}}$. In addition to the standard C library for CDF, they provide a FORTRAN interface and an interface for Perl-the latest addition is support for C\#. As Python is not supported by the NASA team, but is growing in popularity in the space science community we have written a module to handle CDF files.

The C library is made available in Python using ctypes and an object-oriented "thin layer" has been written to provide a Pythonic interface. For example, to open and query a CDF file, the following code is used:

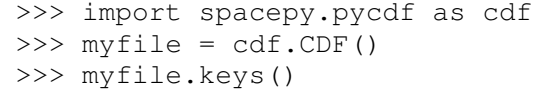

The CDF object inherits from the collections.MutableMapping object and provides the user a familiar 'dictionary-like' interface to the file contents. Write and edit capabilities are also fully supported, further development is being targeted towards the generation of ISTPcompliant CDF files ${ }^{3}$ for the upcoming Radiation Belt Storm Probes (RBSP) mission.

As an example of this use, creating a new CDF from a master (skeleton) CDF has similar syntax to opening one:

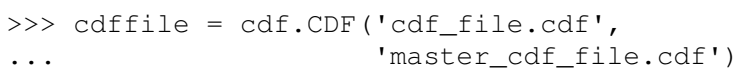

This creates and opens cdf_filename.cdf as a copy of master_cdf_filename.cdf. The variables can then be populated by direct assignment, as one would populate any new object. Full documentation can be found both in the docstrings and on the SpacePy website.

\footnotetext{
2. http://cdf.gsfc.nasa.gov/

3. http://spdf.gsfc.nasa.gov/sp_use_of_cdf.html
}

\section{Radiation belt modeling}

Geosynchronous communications satellites are especially vulnerable to outer radiation belt electrons that can penetrate deep into the system and cause electrostatic charge buildup on delicate electronics. The complicated physics combined with outstanding operational challenges make the radiation belts an area of intense research. A simple yet powerful numerical model of the belts is included in SpacePy in the RadBelt module. This module allows users to easily set up a scenario to simulate, obtain required input data, perform the computation, then visualize the results. The interface is simple enough to allow users to easily include an analysis of radiation belt conditions in larger magnetospheric studies, but flexible enough to allow focused, in-depth radiation belt research.

The model is a radial diffusion model of trapped electrons of a single energy and a single pitch angle. The heart of the problem of radiation belt modeling through the diffusion equation is the specification of diffusion coefficients, source and loss terms. Determining these values is a complicated problem that is tackled in a variety of different ways, from first principles approaches to simpler empirical relationships. The RadBelt module approaches this with a paradigm of flexibility: while default functions that specify these values are given, many are available and additional functions are easy to specify. Often, the formulae require input data, such as the Kp or Dst indices. This is true for the RadBelt defaults. These data are obtained automatically from the OMNI database, freeing the user from the tedious task of fetching data and building input files. This allows simple comparative studies between many different combinations of source, loss, and diffusion models.

Use of the RadBelt module begins with instantiation of an RBmodel object. This object represents a version of the radial diffusion code whose settings are controlled by its various object attributes. Once the code has been properly configured, the time grid is created by specifying a start and stop date and time along with a step size. This is done through the setup_ticks instance method that accepts datetime or Ticktock arguments. Finally, the evolve method is called to perform the simulation, filling the PSD attribute with phase space densities for all $L$ and times specified during configuration. The instance method plot yields a quick way to visualize the results using matplotlib functionality. The example given models the phase space density during the "Hallowe'en" storms of 2003. The results are displayed in figure 4. In the top frame, the phase space density is shown. The white line plotted over the spectrogram is the location of the last closed drift shell, beyond which the electrons escape the magnetosphere. Directly below this frame is a plot of the two geomagnetic indices, Dst and $\mathrm{Kp}$, used to drive the model. With just a handful of lines of code, the model was setup, executed, and the results were visualized.

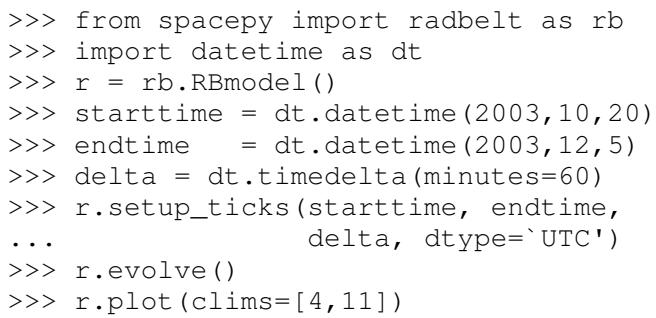



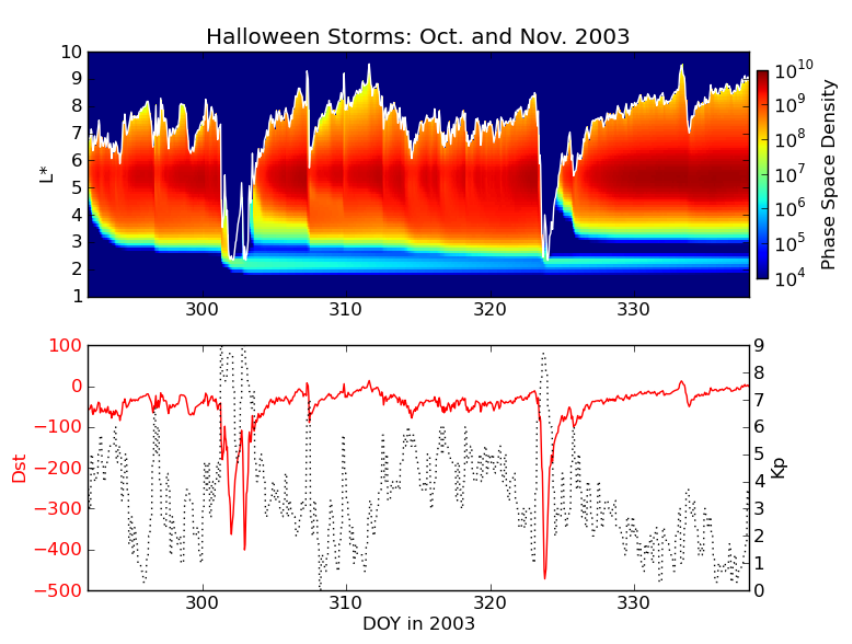

Fig. 4: RadBelt simulation results for the 2003 Hallowe'en storms. The top frame shows phase space density as a function of drift shell and time. The bottom frame shows the geomagnetic Kp and Dst indices during the storm.

\section{Visualizing space weather models}

The Block Adaptive Tree Solar wind Roe-type Upwind Scheme code, or BATS-R-US, is a widely used numerical model in the space science community. It is a magnetohydrodynamic (MHD) code [Pow99], which means it combines Maxwell's equations for electromagnetism with standard fluid dynamics to produce a set of equations suited to solving spatially large systems while using only modest computational resources. It is unique among other MHD codes in the space physics community because of its automatic grid refinement, compile-time selection of many different implementations (including multi fluid, Hall resistive, and nonisotropic MHD), and its library of run-time options (such as solver and scheme configuration, output specification, and much more). It has been used in a plethora of space applications, from planetary simulations (including Earth [Wel10b] and Mars [Ma07]) to solar and interplanetary investigations [Coh09]. As a key component of the Space Weather Modeling Framework (SWMF) [Tot07], it has been coupled to many other space science numerical models in order to yield a true 'sun to mud' simulation suite that handles each region with the appropriate set of governing equations.

Visualizing output from the BATS-R-US code comes with its own challenges. Good analysis requires a combination of two and three dimensional plots, the ability to trace field lines and stream lines through the domain, and the slicing of larger datasets in order to focus on regions of interest. Given that BATS-R-US is rarely used by itself, it is also important to be able to visualize output from the coupled codes used in conjunction. Professional computational fluid dynamic visualization software solutions excel at the first points, but are prohibitively expensive and often leave the user searching for other solutions when trying to combine the output from all SWMF modules into a single plot. Scientific computer languages, such as IDL ${ }^{\mathrm{TM}}$ and MatLab ${ }^{\mathrm{TM}}$, are flexible enough to tackle the latter issue, but do not contain the proper tools required by fluid dynamic applications. Because all of these solutions rely on proprietary software, there are always license fees involved before plots can be made.

The PyBats package of SpacePy attempts to overcome these difficulties by providing a free, platform independent way to read
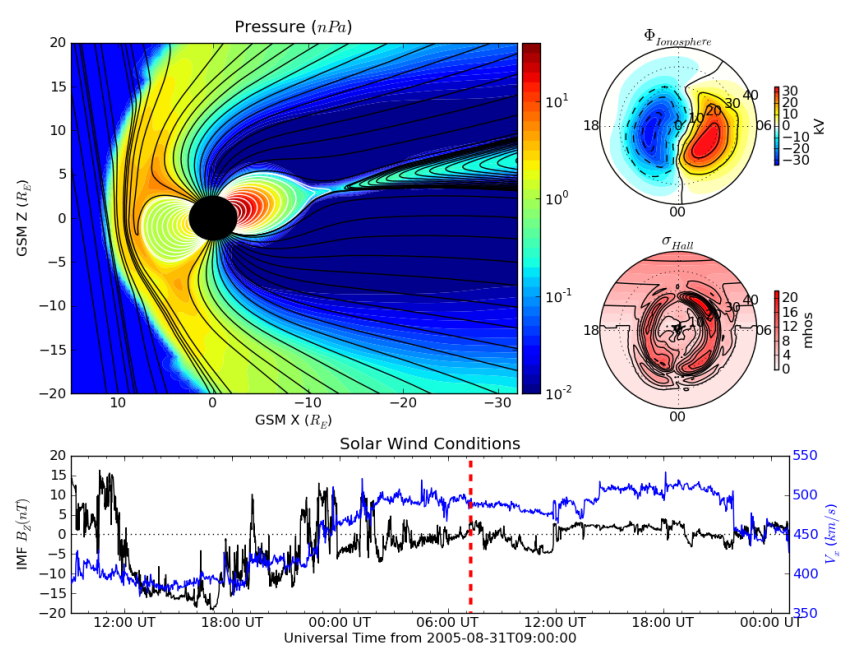

Fig. 5: Typical output desired by users of BATS-R-US and the SWMF. The upper left frame is a cut through the noon-midnight meridian of the magnetosphere as simulated by BATS-R-US at 7:15 UT on September 1, 2005. The dial plots to the left are the ionospheric electric potential and Hall conductivity at the same time as calculated by RIM. Below are the solar wind conditions driving both models.

and visualize BATS-R-US output as well as output from models that are coupled to it. It builds on the functionality of NumPy and matplotlib to provide specialized visualization tools that allow the user to begin evaluating and exploring output as quickly as possible.

The core functionality of PyBats is a set of classes that read and write SWMF file formats. This includes simple ASCII $\log$ files, ASCII input files, and a complex but versatile selfdescriptive binary format. Because many of the codes that are integrated into the SWMF use these formats, including BATS-RUS, it is possible to begin work right away with these classes. Expanded functionality is found in code-specific modules. These contain classes to read and write output files, inheriting from the PyBats base classes when possible. Read/write functionality is expanded in these classes through object methods for plotting, data manipulation, and common calculations.

Figure 5 explores the capabilities of PyBats. The figure is a typical medley of desired output from a basic simulation that used only two models: BATS-R-US and the Ridley Ionosphere Model. Key input data that drove the simulation is shown as well. Creating the upper left frame of figure 5, a two dimensional slice of the simulated magnetosphere saved in the SWMF binary format, would require far more work if the base classes were chosen. The bats submodule expands the base capability and makes short work of it. Relevant syntax is shown below. The file is read by instantiating a Bats $2 d$ object. Inherited from the base class is the ability to automatically detect bit ordering and the ability to carefully walk through the variable-sized records stored in the file. The data is again stored in a dictionary as is grid information; there is no time information for the static output file. Extra information, such as simulation parameters and units, are also placed into object attributes. The unstructured grid is not suited for matplotlib, so the object method regrid is called. The object remembers that it now has a regular grid; all data and grid vectors are now two dimensional arrays. Because this is a computationally expensive step, the regridding is performed to a resolution of 0.25 Earth 
radii and only for a subset of the total domain. The object method contourf, a wrapper to the matplotlib method of the same name, is used to add the pressure contour to an existing axis, ax. The wrapped function accepts keys to the grid and data dictionaries of the Bats2d object to prevent the command from becoming overly verbose. Extra keyword arguments are passed to matplotlib's contourf method. If the original file contains the size of the inner boundary of the code, this is reflected in the object and the method add_body is used to place it in the plot.

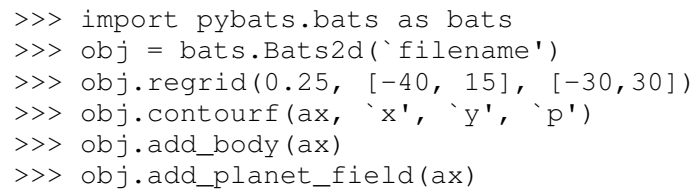

The placement of the magnetic field lines is a strength of the bats module. Magnetic field lines are simply streamlines of the magnetic field vectors and are traced through the domain numerically using the Runge-Kutta 4 method. This step is implemented in $\mathrm{C}$ to expedite the calculation and wrapped using f2py. The Bats $2 \mathrm{~d}$ method add_planet_field is used to add multiple field lines; this method finds closed (beginning and ending at the inner boundary), open (beginning or ending at the inner boundary, but not both), or pure solar wind field lines (neither beginning or ending at the inner boundary) and attempts to plot them evenly throughout the domain. Closed field lines are colored white to emphasize the open-closed boundary. The user is naive to all of this, however, as one call to the method works through all of the steps.

The last two plots, in the upper right hand corner of figure 5 , are created through the code-specific rim module, designed to handle output from the Ridley Ionosphere Model (RIM) [Rid02].

PyBats capabilities are not limited to what is shown here. The Stream class can extract values along the streamline as it integrates, enabling powerful flow-aligned analysis. Modules for other codes coupled to BATS-R-US, including the Ring current Atmosphere interactions Model with Self-Consistent Magnetic field (RAM-SCB, ram module) and the Polar Wind Outflow Model (PWOM, pwom module) are already in place. Tools for handling virtual satellites (output types that simulate measurements that would be made if a suite of instruments could be flown through the model domain) have already been used in several studies. Combining the various modules yields a way to richly visualize the output from all of the coupled models in a single language. PyBats is also in the early stages of development, meaning that most of the capabilities are yet to be developed. Streamline capabilities are currently being upgraded by adding adaptive step integration methods and advanced placement algorithms. Bats $3 \mathrm{~d}$ objects are being developed to complement the more frequently used two dimensional counterpart. A GUI interface is also under development to provide users with a point-and-click way to add field lines, browse a time series of data, and quickly customize plots. Though these future features are important, PyBats has already become a viable free alternative to current, proprietary solutions.

\section{SpacePy in action}

A number of key science tasks undertaken by the SpacePy team already heavily use SpacePy. Some articles in peer-reviewed literature have been primarily produced using the package (e.g.
[Mor10], [Wel10a]). The Science Operations Center for the RBSP mission is also incorporating SpacePy into its processing stream.

The tools described here cover a wide range of routine analysis and visualization tasks utilized in space science. This software is currently available on a non-commercial research license, but the process to release it as free and open-source software is underway. Providing this package in Python makes these tools accessible to all, provides openness in the analysis methods employed in scientific studies and will give access to advanced tools to all space scientists regardless of affiliation or circumstance. The SpacePy team can be contacted at spacepy-info@lanl.gov.

\section{REFERENCES}

[Bos07] D. Boscher, S. Bourdarie, P. O'Brien and T. Guild ONERA-DESP library $v 4.1$, http://irbem.sourceforge.net/, 2007.

[Chr08] C. Chree Magnetic declination at Kew Observatory, 1890 to 1900, Phil. Trans. Roy. Soc. A, 208, 205-246, 1908.

[Coh09] O. Cohen, I.V. Sokolov, I.I. Roussev, and T.I. Gombosi Validation of a synoptic solar wind model, J. Geophys. Res., 113, 3104, doi:10.1029/2007JA012797, 2009.

[Kin05] J.H. King and N.E. Papitashvili Solar wind spatial scales in and comparisons of hourly Wind and ACE plasma and magnetic field data, J. Geophys. Res., 110, A02209, 2005.

[Ma07] Y.J. Ma, A.F. Nagy, G. Toth, T.E. Cravens, C.T. Russell, T.I. Gombosi, J.-E. Wahlund, F.J. Crary, A.J. Coates, C.L. Bertucci, F.M. Neubauer $3 D$ global multi-species Hall-MHD simulation of the Cassini T9 flyby, Geophys. Res. Lett., 34, 2007.

[Mly10] M.G. Mlynczak, L.A. Hunt, J.U. Kozyra, and J.M. Russell III Short-term periodic features observed in the infrared cooling of the thermosphere and in solar and geomagnetic indexes from 2002 to 2009 Proc. Roy. Soc. A, doi:10.1098/rspa.2010.0077, 2010.

[Mor07] S.K. Morley and M.P. Freeman On the association between northward turnings of the interplanetary magnetic field and substorm onset, Geophys. Res. Lett., 34, L08104, 2007.

[Mor10] S.K. Morley, R.H.W. Friedel, E.L. Spanswick, G.D. Reeves, J.T Steinberg, J. Koller, T. Cayton, and E. Noveroske Dropouts of the outer electron radiation belt in response to solar wind stream interfaces: Global Positioning System observations, Proc. Roy. Soc. A, doi:10.1098/rspa.2010.0078, 2010.

[Pow99] K. Powell, P. Roe, T. Linde, T. Gombosi, and D.L. De Zeeuw A solution-adaptive upwind scheme for ideal magnetohydrodynamics, J. Comp. Phys., 154, 284-309, 1999.

[Qin07] Z. Qin, R.E. Denton, N. A. Tsyganenko, and S. Wolf Solar wind parameters for magnetospheric magnetic field modeling, Space Weather, 5, S11003, 2007.

[Rid02] A.J. Ridley and M.W. Liemohn A model-derived storm time asymmetric ring current driven electric field description J. Geophys. Res., 107, 2002.

[Tot07] Toth, G., D.L.D. Zeeuw, T.I. Gombosi, W.B. Manchester, A.J. Ridley, I.V. Sokolov, and I.I. Roussev Sun to thermosphere simulation of the October 28-30, 2003 storm with the Space Weather Modeling Framework, Space Weather, 5, S06003, 2007.

[Vai09] R. Vainio, L. Desorgher, D. Heynderickx, M. Storini, E. Fluckiger, R.B. Horne, G.A. Kovaltsov, K. Kudela, M. Laurenza, S. McKennaLawlor, H. Rothkaehl, and I.G. Usoskin Dynamics of the Earth's Particle Radiation Environment, Space Sci. Rev., 147, 187--231, 2007.

[Wel10a] D.T. Welling, and A.J. Ridley Exploring sources of magnetospheric plasma using multispecies MHD, J. Geophys. Res., 115, 4201, 2010

[Wel10b] D.T. Welling, V. Jordanova, S. Zaharia, A. Glocer, and G. Toth The effects of dynamic ionospheric outflow on the ring current, Los Alamos National Laboratory Technical Report, LA-UR 10-03065, 2010.

[Wil09] J.A. Wild, E.E. Woodfield, and S.K. Morley, On the triggering of auroral substorms by northward turnings in the interplanetary magnetic field, Ann. Geophys., 27, 3559-3570, 2009. 